\title{
Are the Symptoms of Late Urogenital Toxicity After Radiotherapy for Localized Prostate Cancer Transient or Do They Worsen Over Time?
}

Vesna V. Stankovic ( $\square$ vesna.stankovic0704@gmail.com )

Institute of Oncology and Radiology of Serbia

Zoran M. Bukumiric

University of Belgrade

Jelena D. Stanic

Institute of Oncology and Radiology of Serbia

Katarina V. Kopcalic

Institute of Oncology and Radiology of Serbia

Nenad M. Milosevic

Institute of Oncology and Radiology of Serbia

\section{Tanja V. Raonic-Stevanovic}

Institute of Oncology and Radiology of Serbia

\section{Marina R. Nikitovic}

University of Belgrade

\section{Research Article}

Keywords: prostate cancer, 3D conformal radiotherapy, late toxicity, genitourinary toxicity

Posted Date: July 30th, 2021

DOI: https://doi.org/10.21203/rs.3.rs-745039/v1

License: (a) This work is licensed under a Creative Commons Attribution 4.0 International License.

Read Full License 


\section{Abstract}

Our aim is to estimate incidence and prevalence rates as well as overall changes in the degree of late urogenital toxicity (UGT) over time in patients treated with 3D conformal radiotherapy (3DCRT) for localized prostate cancer (PC) and to explored possible predictive factors for late UGT.

Ninety-four patients with localized PC, treated with 3DCRT with a total dose of 72Gy/2Gy/daily, were examined in this study. The median follow-up was 66 months. Many individual and clinical characteristics were explored as possible predictive factors for late UGT.

Incidences of late UGT grade $\geq 1, \geq 2$ and grade 3 were $64.89 \%$ (61/94), 22.34\% (21/94) and 7.4\% (7/94), respectively. The prevalence rates of late UGT grade $\geq 1$, grade $\geq 2$ and grade 3 were $26.6 \%-36.3 \%$, 3.2$13.3 \%$ and $1.06 \%-7.89 \%$, respectively. Statistically significant positive trend was found favoring an increase in the degree of late UGT over time $(b=0.028 ; p<0.001)$. In the MVA, previous abdominal and pelvic surgery (PAPS) and acute UGT were significantly associated with late UGT grade $\geq 1(p=0.007)$ and $(p=0.013)$, respectively.

We can conclude that the degree of late UGT increases over time. PAPS and the occurrence of any grade of acute UGT have a significant influence on the development of any grade of late UGT.

\section{Introduction}

Technological advances from the end of the last century made it possible to introduce 3D conformal radiotherapy (3DCRT) into everyday clinical practice with the aim of increasing tumor control by dose escalation and reduction of urogenital toxicity (UGT) and gastrointestinal toxicity (GIT). However, randomized trials have shown no significant difference in the occurrence of acute or late UGT between conventional radiotherapy and 3DCRT [1, 2]. More recently, intensity-modulated radiotherapy (IMRT), volumetric modulated arc therapy (VMAT) and precise image guidance radiotherapy (IGRT) were implemented to improve the conformality of the dose distribution, and thereby facilitate dose escalation for localized prostate cancer (PC) patients. Despite the advanced technique, the results of phase 3 doseescalation trials suggest that there were no significant differences between 3DCRT and IMRT for acute or late G2 + or G3 + UGT [3]. Moderate hypofractionation is safe and effective, but longer-term data are still lacking.

In addition to the dose-volume effect reported in the literature, other factors that may affect the incidence of acute and late UGT such as age, smoking, hormonal therapy, diabetes mellitus (DM), certain drugs, genetic and other biological markers have been mentioned in the literature [4-12].

The link between the acute and late effects of radiotherapy (RT) in PC has been observed by several investigators [13], and its existence confirmed by the results obtained in the Medical Research Council RT01 TRIAL [14]. 
Late side-effects of RT for the PC can be irreversible and may decrease the quality of life (QoL) but Schmid et al. demonstrates that the majority of late GIT and UGT symptoms after primary 3DCRT for PC are temporary [15].

In a previously published study [16] we found a statistically significant link of any grade of acute UGT with any grade of late UGT by MVA analysis. After considering the results obtained in our previous study, we wanted to examine whether, after a longer follow-up, this link would exist and whether clinical and patient-related factors could influence the occurrence of late UGT and if so, which ones.

Furthermore, since we observed that symptoms of UGT may be temporary and may occur during followup, we wanted to investigate whether these symptoms worsen over time, remain of the same intensity, or decline in their degree. Therefore, we decided to evaluate changes in the degree of late UGT over time.

For this reason, we conducted a longitudinal study among localized PC patients.

\section{Results}

The median follow-up for all 94 patients was 66 (7-101) months. Tumor characteristics such as T-stage, initial PSA, grading and Gleason Score are shown in Table 1.

Table 1

Tumor characteristics

\begin{tabular}{|ll|}
\hline Tumor characteristics & \\
\hline Mean PSA+/-SD, ng /ml(range) & $9.884+/-3.186(4.14-17.4)$ \\
\hline Gleason score & \\
$\leq 6$ & $68(72.3 \%)$ \\
\hline 7 & $26(27.7 \%)$ \\
\hline $7(3+4)$ & $10(10.7 \%)$ \\
\hline $7(4+3)$ & $16(17 \%)$ \\
\hline Risk categories & \\
\hline Low risk & $53(56.4 \%)$ \\
\hline Intermediate risk & $41(43.6 \%)$ \\
\hline TNM stage & \\
\hline I stage (T1NOM0) & $50(53.2 \%)$ \\
\hline II stage (T2NOM0) & $44(46.8 \%)$ \\
\hline
\end{tabular}


Description of individual characteristics of patients, clinical and treatment characteristics and dosimetric parameters are shown in Table 2 and Table 3.

Table 2

UVA analysis according to individual and clinical characteristics

\begin{tabular}{|llll|}
\hline variable & description & \multicolumn{2}{l|}{ Univariate analysis* } \\
\cline { 4 - 4 } & & b & p \\
\hline Mean age \pm standard deviation (years) & $71,32+/-5,401$ & 0,012 & 0,168 \\
\hline Current smoking status (yes) & $13(13.8 \%)$ & 0,053 & 0,703 \\
\hline Alcohol consummation (yes) & $33(35.1 \%)$ & $-0,014$ & 0,892 \\
\hline Cardiovascular disease (yes) & $67(71.3 \%)$ & $-0,021$ & 0,846 \\
\hline Diabetes mellitus type II (yes) NIDDM & $13(13.8 \%)$ & 0,276 & 0,043 \\
\hline Previous abdominal or pelvic surgery (yes) PAPS & $32(34.0 \%)$ & 0,246 & 0,013 \\
\hline Antiagregation therapy (yes) & $41(43.6 \%)$ & $-0,028$ & 0,768 \\
\hline Anticoagulant therapy (yes) & $3(3.2 \%)$ & 0,316 & 0,242 \\
\hline Arterial hypertension (HTA) (yes) & $62(66.0 \%)$ & $-0,116$ & 0,250 \\
\hline Angina pectoris (yes) & $23(24.5 \%)$ & $-0,094$ & 0,397 \\
\hline Cerebrovascular infarction (CVI) (yes) & $4(4.3 \%)$ & $-0,134$ & 0,571 \\
\hline Acute myocardial infarction (AIM) (yes) & $10(10.6 \%)$ & 0,239 & 0,126 \\
\hline Neoadjuvant hormonal therapy (yes) & $16(17.0 \%)$ & $-0,171$ & 0,175 \\
\hline $\begin{array}{l}\text { Depending on the type of variables and the normality of the distribution, descriptiveness is presented } \\
\text { as N (\%), as } \pm \text { SD or median (min-max). }\end{array}$ & & \\
\hline $\begin{array}{l}\text { * Ordinal multilevel regression models with a degree of late UGT as the dependent variable adjacent } \\
\text { during observation. }\end{array}$ & & & \\
\hline
\end{tabular}


Table 3

UVA analysis according to treatment characteristics

\begin{tabular}{|llll|}
\hline variable & description & \multicolumn{2}{l}{ Univariate analysis* } \\
\cline { 3 - 4 } & & $\mathbf{b}$ & $\mathbf{p}$ \\
\hline Dose volume group: Prostate only & $47(50.0 \%)$ & $-0,0636$ & 0,505 \\
\hline Dose volume group: Prostate + SV & $47(50.0 \%)$ & $-0,0010$ & 0,656 \\
\hline Median Prostate volume (ccm) & $54.5(19.9-108.5)$ & 0,0005 & 0,142 \\
\hline Median Bladder volume (ccm) & $148.8(64.1-952.6)$ & 0,0013 & 0,310 \\
\hline Median Rectum volume (ccm) & $82.4(34.3-260.3)$ & $-0,0189$ & 0,874 \\
\hline Median D max b (Gy) & $72.51(71.3-74.1)$ & $-0,0021$ & 0,614 \\
\hline Median D mean b (Gy) & $56.3(16.7-68.9)$ & $-0,0026$ & 0,244 \\
\hline Median V50 b (\%) & $67 .(14.0-100.0)$ & $-0,0028$ & 0,248 \\
\hline Median V60 b (\%) & $50.4(10.0-90.1)$ & $-0,0029$ & 0,264 \\
\hline Median V65 b (\%) & $41.7(7.7-83.0)$ & $-0,0034$ & 0,368 \\
\hline Median V70 b (\%) & $20.3(3.9-66.5)$ & $-0,0740$ & 0,520 \\
\hline Acute UGT (no of patients, \%) & $25(30.1 \%)$ & $-0,0971$ & 0,548 \\
\hline $\begin{array}{l}\text { Depending on the type of variables and the normality of the distribution, descriptiveness is presented } \\
\text { as N (\%), as } \pm \text { SD or median (min-max). }\end{array}$ & & \\
\hline $\begin{array}{l}\text { * Ordinal multilevel regression models with a degree of late UGT as the dependent variable adjacent } \\
\text { during observation. }\end{array}$ & & & \\
\hline
\end{tabular}

During the follow-up, Late UGT, max grade $\geq 1, \geq 2$ and 3 developed in 61/94(64.89\%), 21/94 (22.34\%) and $7 / 94(7.4 \%)$ patients, respectively.

Thirty-three of 94 patients (35.11\%) didn't show symptoms of late UGT during a follow-up.

The estimated median time of the onset of symptoms of late UGT grade $\geq 1$ is 18 months $(95 \% \mathrm{Cl} 4.8-$ 31.2). (Fig. 1)

Late UGT grade 3 developed in 7 patients: in 3 of them after 3, 18 and 54 months since the follow-up and it lasted for 9, 6 and 18 months, whereas in the other 4 patients symptoms of late UGT grade 3 were recorded at the last check-up: $36,42,72$ and 72 months after the completion of the radiation treatment.

Prevalence rate of late UGT grade $\geq 1$ was $26.9 \%, 28.25 \%, 26,6 \%, 31.3 \%, 33.3 \%$ and $36,8 \%$ after $12,24,36$, 48,60 and 72 months, respectively while prevalence rate of late UGT grade $\geq 2$ was $3.2 \%, 7,6 \%, 5.6 \%$, 
$8.4 \%, 13.3 \%$, and $10,5 \%$ and grade 3 was $1.06 \%, 1.07 \%, 1.11 \%, 1.20 \%, 3.33 \%, 7.89 \%$ after $12,24,36,48,60$ and 72 months, respectively.

The Distribution of the prevalence rate of late UGT according to the grade at each time point during the follow-up is shown at Table 4.

Table 4

The Distribution of the prevalence rate of late UGT according to the grade, at each time point during the follow-up

\begin{tabular}{|llllllllllllllll|l|}
\hline Late UGT & \multicolumn{10}{l|}{ Months after the end of RT } \\
\cline { 2 - 5 } & $\mathbf{0}$ & $\mathbf{3}$ & $\mathbf{6}$ & $\mathbf{1 2}$ & $\mathbf{1 8}$ & $\mathbf{2 4}$ & $\mathbf{3 0}$ & $\mathbf{3 6}$ & $\mathbf{4 2}$ & $\mathbf{4 8}$ & $\mathbf{5 4}$ & $\mathbf{6 0}$ & $\mathbf{7 2}$ & $\mathbf{8 4}$ & $\mathbf{9 6}$ \\
\hline 0 & 91 & 69 & 69 & 68 & 65 & 66 & 67 & 66 & 65 & 57 & 49 & 40 & 24 & 10 & 1 \\
\hline 1 & 2 & 20 & 22 & 22 & 24 & 19 & 19 & 19 & 15 & 19 & 18 & 12 & 10 & 1 & 2 \\
\hline 2 & 1 & 4 & 2 & 2 & 3 & 6 & 6 & 4 & 7 & 6 & 6 & 6 & 1 & 1 & 0 \\
\hline 3 & 0 & 1 & 1 & 1 & 1 & 1 & 0 & 1 & 2 & 1 & 1 & 2 & 3 & 0 & 0 \\
\hline $\begin{array}{l}\text { Total No of } \\
\text { pts }\end{array}$ & 94 & 94 & 94 & 93 & 93 & 92 & 92 & 90 & 89 & 83 & 74 & 60 & 38 & 12 & 3 \\
\hline
\end{tabular}

All changes in the degree of late UGT at each time point for each patient during the follow-up are shown in Fig. 2.

As this is a partially retrospective study, we did not have any pre-treatment symptoms recorded in the medical history, so we took the symptoms recorded at the first check-up during the first week of radiation treatment.

The changes in the degree of late UGT at particular time points for each patent were examined with a linear mixed effects modeling approach and it was found that there is a statistically significant positive trend for an increase in the overall degree of late UGT over time $(b=0.028 ; p<001)$. (Fig. 2$)$

Individual characteristics of patients, clinical and treatment characteristics and dosimetric parameters which are investigated as possible predictors of late UGT by univariate analysis are shown in Table 2 and Table 3. Statistically significant predictors for late UGT according to the UVA were DM type II $(p=0.043)$, PAPS $(p=0.013)$ and acute UGT $(p=0.014)$.

Toxicity predictors from univariate analysis which were statistically significant were included in the multivariate multilevel ordinal regression model. In a multivariate multilevel ordinal regression model, PAPS $(p=0.007)$ and acute UGT during radiotherapy $(p=0.013)$ were statistically significantly associated with a higher degree of late UGT (Table 5). 
Table 5

Predictors of late UGT grade $\geq 1$

\begin{tabular}{|c|c|c|}
\hline \multirow[t]{2}{*}{ Variable } & \multicolumn{2}{|c|}{ Multivariate analysis* } \\
\hline & b & $\mathbf{p}$ \\
\hline Diabetes mellitus & 0,220 & 0,090 \\
\hline Previous abdominal or pelvic surgery & 0,256 & 0,007 \\
\hline Acute UGT & 0,232 & 0,013 \\
\hline
\end{tabular}

\section{Discussion}

Despite the introduction of more advanced techniques such as IMRT, VMAT and IGRT in clinical practice, reductions in acute and late toxicity were observed only in rectal symptoms [17]. On the other hand, results of phase 3 dose-escalation trials suggest that there were no significant differences with 3DCRT or IMRT for acute or late G2 + or G3 + UGT [3]. In addition to the RT technique, other factors are involved in the occurrence of symptoms of UGT. Understanding the impact of dosimetric/clinical factors on urinary symptom after RT is crucial to decision-making process for a more tailored treatment and patient care [18].

In our prior publication we estimated the incidence of acute and late UGT after a median follow-up of 27 months and influence of individual/clinical factors on late UGT [16]. Since we know that the influence of the follow-up length is important because some symptoms appear later, we decided to continue our investigation with update the results. After median follow-up of 66 months we have got new results: thirty-three of 94 patients (35.11\%) showed no symptoms of late UGT.

To overcome the limitations in toxicity reporting by using incidence rates, other approaches were proposed in the literature.

Schmid et al. suggests that the combination of incidence and prevalence rates provides a more comprehensive view on the complex issue of late side effects because incidence rates may lead to misinterpretation or overestimation. Their study demonstrates that the majority of late GIT and UGT after primary external beam RT for PC are temporary [15].

In contrast, Olson et al. [19] found that the temporal pattern of symptom occurrence varied, but the prevalence generally changed moderately over time.

In our study the prevalence rate of late UGT grade $\geq 1$ increase over time while the prevalence rate of late UGT grade $\geq 2$ fluctuates. 
The increase and decrease of prevalence rate at different time points during follow-up suggests that in some patients the symptoms may be diminished and/or be temporary, while in other patients, symptoms may be developed "de novo" or pre-existing symptoms may have worsened.

Therefore, we wanted to take into account all changes in the degree of late UGT at all-time points for each patient, in order to determine how the overall grade of late UGT changes over time.

Having in mind the fluctuation of the prevalence of late UGT symptoms, we expected a decrease in the degree of late UGT symptoms, which would mean that these symptoms are temporary and that they may heal over time.

Surprisingly, we found that there is a statistically significant positive trend for an increase in the overall degree of late UGT over time ( $b=0.028 ; p<001)$ (Fig. 2). Based on this result, we can conclude that if we follow these patients long enough (longer than 5 years), the degree of late UGT will be higher.

As far as we know, this is the first time in the literature that late toxicity is assessed taking into account all changes in the degree of late UGT at all -time points for each patient, in order to determine how the overall degree of late UGT changes over time.

The second goal of our study was to estimate the possible influence of individual and clinical characteristics on the occurrence of late UGT.

In RADAR study, several parameters affecting the development of specific urinary symptoms after prostate cancer radiotherapy were identified. Baseline symptoms and non-insulin-dependent diabetes mellitus (NIDDM) were found to have sustained impacts across all time point, while age and PC1 (PC1 has high correlation to the mean dose) were repeated in more than one time point. Other associations found to be significant are not repeated at different time points suggesting poorer or temporary impacts [4].

As this is a partially retrospective study, we did not have any pre-treatment symptoms recorded in the medical history so we could not examine the correlation between baseline symptoms and the occurrence of late UGT.

In the current study NIDDM in UVA was statistically significant $(p=0.043)$ but in MVA was not significant $(p=0.09)$. Similar results we got in our former study: in UVA, NIDDM was statistically significant $(p=$ $0.028)$ but in MVA was border line significant $(p=0.056)[16]$.

In the literature, we found studies which reported a correlation between PAPS and the symptoms of late GIT [20], but we did not find any which reported correlations between PAPS and the advent of late UGT.

The authors who found a correlation between previous surgery and GIT symptoms assumed that the inflammatory response could increase due to surgery even though the surgical injury was outside the irradiated volume and therefore radiation sensitivity increases. It has also been observed that cytokine 
expression increases due to surgery, which could lead to a more pronounced inflammatory response of the gastrointestinal mucosa to radiation [21, 22].

Christensen et al. [23] observed that the expression of IL1 and IL6 was associated with an increased probability for acute UGT and GIT, respectively.

In contrast, Bedini et al. [24] in their study showed that IL-1b and IL-6 were fairly undetectable in most patients and presented with very low variability among patients. This preliminary study identified a correlation between CCL2 levels at the end of radiotherapy and basal CCL2, age, and previous abdominal/pelvic surgery (PAPS), suggesting a different response to RT in older patients and in patients with pretreatment abdominal surgery.

In our prior study PAPS was a statistically significant factor for grade $\geq 2$ of acute UGT $(p=0.012)$ but not for late UGT $(=0.108)[16]$. Interestingly, in our current study PAPS was a statistically significant factor $(p=0.007)$ for late UGT grade $\geq 1$. To our knowledge, we are so far the only one to report a statistically significant association between PAPS and acute and late UGT. The relationship between acute and late effects of RT remains controversial.

It was traditionally thought that there was no link between the acute reaction to radiation exposure and the late effects in other organs and tissues, and that acute and late toxicity were the consequences of different independent pathological mechanisms. It has been shown that the acute and late toxicity do not share a similar set of predictors $[25,26]$. More aggressive RT protocols can result in aggravation, i.e. an increase in severity and duration, of acute radiation effects [27]. The non-healing response can progress directly into a late effect. In other examples of consequential late effects, there is clinical healing of acute effects and a symptom-free period before the independent development of late effects in the same tissue [26].

In the Medical Research Council RT01 TRIAL [14], acute bladder symptoms correlated with the presence of all late bladder symptoms scores but only with a change in the score for the symptoms of urinary frequency and hematuria. This therefore suggests that patients with symptoms before treatment will probably suffer acute bladder toxicity during treatment and may develop late symptoms.

Pinkawa et al. [28] found a strong dependence of urinary/bowel QoL after radiotherapy on urinary/bowel QoL before radiotherapy. In contrast to absolute scores, QoL score changes (relative to baseline scores) did not correlate with pretreatment scores. They concluded that long-term changes could be well predicted by acute changes.

In our current study applying the multivariate multilevel ordinal regression model we found, apart from PAPS, acute UGT during radiotherapy to be statistically significantly associated with a higher degree of late UGT $(p=0.013)$. The conclusions coincide with the results from our previous study $(p=0.024)$.

Based on the obtained results, we can conclude that in the examined group the degree of late UGT increased with time. PAPS is a statistically significant predictive factor for the onset of late UGT 
symptoms. There is a statistically significant correlation between the frequency and the severity of symptoms of acute and late UGT.

\section{Methods}

Patients were treated at a single institution with 3DCRT in the period between September 2009 and September 2013 and a total 94/225 patients seen in this period have been included. Study criteria and treatment schedule have already been reported $[16,29]$. Patients with histologically proven, localized PC with low or intermediate risk, were included. Patients with estimated risk of lymph node involvement > $15 \%$ according to Roach formula and with signs of lymph-nodes positive on CT (computerized tomography) / MRI (magnetic resonance imaging) and with previous pelvic irradiation were excluded. Neo-adjuvant hormonal therapy was not an exclusion criterion. Informed consent was obtained from participants in the study and the institution's ethics committee approved the study.

Patients were divided into two dose volume groups according to the estimated risk of seminal vesicle involvement according to the Roach formula [30]. In the first dose volume group CTV (clinical target volume) included the whole prostate. In the second dose volume group CTV1 encompassed the whole prostate with the entire seminal vesicle and in second phase CTV2 encompassed the whole prostate only. The dose that was prescribed to the ICRU reference volume covered at least $95 \%$ of PTV (planning target volume): $72 \mathrm{~Gy}$ to PTV, 66Gy to PTV1 and 6Gy to PTV2. All patients received total dose of 72Gy/2Gy daily fraction. The safety margin around the CTV and CTV1 were defined according our institutional protocol $[16,29]$. Bladder and rectum dose constraints were taken from Emmami and coworkers first [31]. Since the end of 2010 we have used Quantec recommendations for dose limitation [32].

After the completion of RT patients were seen every 3 months during the first year, then every 6 months and after 5 years annually. Follow ups were done until the occurrence of death or the end of the study (30.4.2018). All symptoms of late UGT were recorded during follow up visits and graded according to the EORTC/RTOG scoring scale slightly modified by Peters and coworkers [25].

In our previously published study [16] our primary goal was the assessment of acute and late UGT after a median follow-up of 27 months while the second was the estimation of the possible influence of individual/clinical characteristics on the occurrence of acute and late UGT.

Later, we continued to monitor these patients and decided to re-evaluate late UGT after a median followup of 66 months and determine whether the same or some other individual and clinical factors influence the onset of late UGT.

In our previous study we estimated incidence of acute and late UGT only. Beside the incidence of late UGT defined as maximal grade of any symptoms at any times during follow-up, we decided to estimate the median time of the onset of late UGT grade $1 \leq$ and a prevalence rate for every grade of late UGT at each time point during the follow-up. In addition, we wanted to estimate whether the grade of late UGT 
worsened over time or whether the symptoms were temporary, taking into account all changes in the degree of late UGT at each time point for each patient since the last check-up after the end of RT.

\section{Statistical analyses}

Depending on the type of variables and the normality of the distribution, results were presented as frequency (percent), median (range) and mean \pm standard deviation. Changes in the degree of late UGT during the follow-up were examined with a linear mixed effects modeling approach using R package Ime4 version 1.1-23. For the modeling of the relation of the ordinal dependent variable (late UGT) in repeated measurements with potential predictors, a ordinal regression was used. Toxicity predictors from univariate analyzes which were statistically significant were included in the multivariate ordinal regression model. All p-values less than 0.05 were considered significant. Statistical data analysis was performed using IBM SPSS Statistics 22 (IBM Corporation, Armonk, NY, USA) and R-3.6.3 software (The R Foundation for Statistical Computing, Vienna, Austria).

\section{Declarations}

\section{Acknowledgments}

The authors acknowledge the support from the Ministry of Education, Science and Technological Development of the Republic of Serbia (No 451-03-68/2020-14/200043).

\section{Author contributions statement}

VS: conceived of the presented idea, designed the study, wrote the main manuscript text, and conduct a comprehensive search of literature.

ZB: analyzed and interpreted obtained data, verified the analytical methods, performed statistical analysis of the obtained results.

JS: enrolled patients in the study, aided in interpreting the results, worked on the manuscript, and participated in writing the manuscript.

KK: enrolled patients in the study, aided in interpreting the results, worked on the manuscript and contributed to the final version of the manuscript.

NM: contributed to the interpretation of the results and performed the calculations.

TRS: analysis, drafted the manuscript and designed the figures, and critically revised the manuscript.

MN: discussed the results, contributed to the final manuscript, and critically revised the manuscript.

All authors read and approved the final manuscript. 
Not applicable.

\section{Conflicts of interest}

The authors declare no potential conflict of interests with respect to the research, authorship, and or publications of this article.

\section{Availability of data and material}

The datasets used and/or analyzed during the current study are available from the corresponding author on reasonable request.

\section{Ethics approval}

This study was performed in line with the principles of the Declaration of Helsinki. Approval was granted by the Ethics Committee of University of Belgrade, Serbia (20.11.2013. No 29/XI-10).

\section{References}

1. Dearnaley, D. P., et al. Comparison of radiation side-effects of conformal and conventional radiotherapy in prostate cancer: a randomised trial. The Lancet, 353(9149), 267-272 (1999).

2. Morris, D. E., et al. Evidence-based review of three-dimensional conformal radiotherapy for localized prostate cancer: an ASTRO outcomes initiative. International Journal of Radiation Oncology* Biology* Physics, 62(1), 3-19 (2005).

3. Michalski, J. M., et al. Preliminary toxicity analysis of 3-dimensional conformal radiation therapy versus intensity modulated radiation therapy on the high-dose arm of the Radiation Therapy Oncology Group 0126 prostate cancer trial. International Journal of Radiation Oncology* Biology* Physics, 87(5), 932-938 (2013).

4. Yahya, N., et al. Dosimetry, clinical factors and medication intake influencing urinary symptoms after prostate radiotherapy: An analysis of data from the RADAR prostate radiotherapy trial. Radiotherapy and Oncology, 116(1), 112-118 (2015).

5. Byrne, K., et al. Late genitourinary toxicity outcomes in 300 prostate cancer patients treated with dose-escalated image-guided intensity-modulated radiotherapy. Clinical Oncology, 29(9), 617-625 (2017).

6. Solanki, A. A., \& Liauw, S. L. Tobacco use and external beam radiation therapy for prostate cancer: influence on biochemical control and late toxicity. Cancer, 119(15), 2807-2814 (2013).

7. Kalakota, K., \& Liauw, S. L. Toxicity after external beam radiotherapy for prostate cancer: an analysis of late morbidity in men with diabetes mellitus. Urology, 81(6), 1196-1201 (2013).

8. Hall, W. H., Jani, A. B., Ryu, J. K., Narayan, S., \& Vijayakumar, S. The impact of age and comorbidity on survival outcomes and treatment patterns in prostate cancer. Prostate Cancer and Prostatic Diseases, 8 (1), 22-30 (2005). 
9. Valicenti, R. K., et al. RTOG 94-06: Is the addition of neoadjuvant hormonal therapy to dose-escalated 3D conformal radiation therapy for prostate cancer associated with treatment toxicity?. International Journal of Radiation Oncology* Biology* Physics, 57(3), 614-620 (2003).

10. De Langhe, S., et al. Acute radiation-induced nocturia in prostate cancer patients is associated with pretreatment symptoms, radical prostatectomy, and genetic markers in the TGF $\beta 1$ gene. International Journal of Radiation Oncology* Biology* Physics, 85(2), 393-399 (2013).

11. Kopcalic, K., et al. Association between miR-21/146a/155 level changes and acute genitourinary radiotoxicity in prostate cancer patients: A pilot study. Pathology-Research and Practice, 215(4), 626631 (2019).

12. Stanojković, T. P., et al. Evaluation of cytokine expression and circulating immune cell subsets as potential parameters of acute radiation toxicity in prostate cancer patients. Scientific reports, 10(1), 1-10 (2020).

13. Jereczek-Fossa, B. A., et al. Correlation between acute and late toxicity in 973 prostate cancer patients treated with three-dimensional conformal external beam radiotherapy. International Journal of Radiation Oncology* Biology* Physics, 78(1), 26-34 (2010).

14. Barnett, G. C., De Meerleer, G., Gulliford, S. L., Sydes, M. R., Elliott, R. M., \& Dearnaley, D. P. The impact of clinical factors on the development of late radiation toxicity: results from the Medical Research Council RT01 trial (ISRCTN47772397). Clinical Oncology, 23(9), 613-624 (2011).

15. Schmid, M. P., et al. Late gastrointestinal and urogenital side-effects after radiotherapy-Incidence and prevalence. Subgroup-analysis within the prospective Austrian-German phase II multicenter trial for localized prostate cancer. Radiotherapy and Oncology, 104(1), 114-118 (2012).

16. Stankovic, V., et al. Acute and late genitourinary toxicity after 72 Gy of conventionally fractionated conformal radiotherapy for localised prostate cancer: impact of individual and clinical parameters. Clinical Oncology, 28(9), 577-586 (2016).

17. Koper P.C., et al. Acute morbidity reduction using 3DCRT for prostate carcinoma: a randomized study. Int J Radiat Oncol Biol Phys, 43:727-34 (1999).

18. Rancati, T., Palorini, F., Cozzarini, C., Fiorino, C., \& Valdagni, R. Understanding urinary toxicity after radiotherapy for prostate cancer: first steps forward. Tumori Journal, 103(5), 395-404 (2017).

19. Olsson, C. E., et al. Patient-reported genitourinary toxicity for long-term prostate cancer survivors treated with radiation therapy. British journal of cancer, 108(10), 1964-1970 (2013).

20. Valdagni, R., et al. Increasing the risk of late rectal bleeding after high-dose radiotherapy for prostate cancer: the case of previous abdominal surgery. Results from a prospective trial. Radiotherapy and Oncology, 103(2), 252-255 (2012).

21. Ong, Z. Y., et al. Pro-inflammatory cytokines play a key role in the development of radiotherapyinduced gastrointestinal mucositis. Radiation oncology, 5(1), 1-8 (2010).

22. Kalff, J. C., et al. Intra-abdominal activation of a local inflammatory response within the human muscularis externa during laparotomy. Annals of surgery, 237(3), 301, (2003). 
23. Christensen, E., et al. Longitudinal cytokine expression during IMRT for prostate cancer and acute treatment toxicity. Clinical Cancer Research, 15(17), 5576-5583 (2009).

24. Bedini, N., et al. Evaluation of mediators associated with the inflammatory response in prostate cancer patients undergoing radiotherapy. Disease markers, 2018 (2018).

25. Peeters, S. T., et al. Acute and late complications after radiotherapy for prostate cancer: results of a multicenter randomized trial comparing $68 \mathrm{~Gy}$ to $78 \mathrm{~Gy}$. International Journal of Radiation Oncology* Biology* Physics, 61(4), 1019-1034(2005).

26. Dörr, W., \& Hendry, J. H. Consequential late effects in normal tissues. Radiotherapy and oncology, 61(3), 223-231 (2001).

27. Peters, L. J., Ang, K. K., \& Thames, H. D. Accelerated fractionation in the radiation treatment of head and neck cancer-a critical comparison of different strategies. Acta oncologica, 27(2), 185-194 (1988).

28. Pinkawa, M., et al. Consequential late effects after radiotherapy for prostate cancer-a prospective longitudinal quality of life study. Radiation Oncology, 5(1), 1-9 (2010).

29. Stankovic, V., Nikitovic, M., Pekmezovic, T., Pekmezovic, D., Kisic Tepavcevic, D., \& Stefanovic Djuric, A. Toxicity of the lower gastrointestinal tract and its predictive factors after $72 \mathrm{~Gy}$ conventionally fractionated 3D conformal radiotherapy of localized prostate cancer. J. BUON, 21(5), 1224-1232 (2016).

30. Roach M. Re: The use of prostate specific antigen, clinical stage and Gleason score to predict pathological stage in men with localized prostate cancer. J Urol. 150(6):1923-4 (1993).

31. Emami, B., et al. Tolerance of normal tissue to therapeutic irradiation. Int J Radiat Oncol Biol Phys, 21, 109-122 (1991).

32. Bentzen, S. M., et al. Quantitative Analyses of Normal Tissue Effects in the Clinic (QUANTEC): an introduction to the scientific issues. International Journal of Radiation Oncology* Biology* Physics, 76(3), S3-S9. (2010).

\section{Figures}




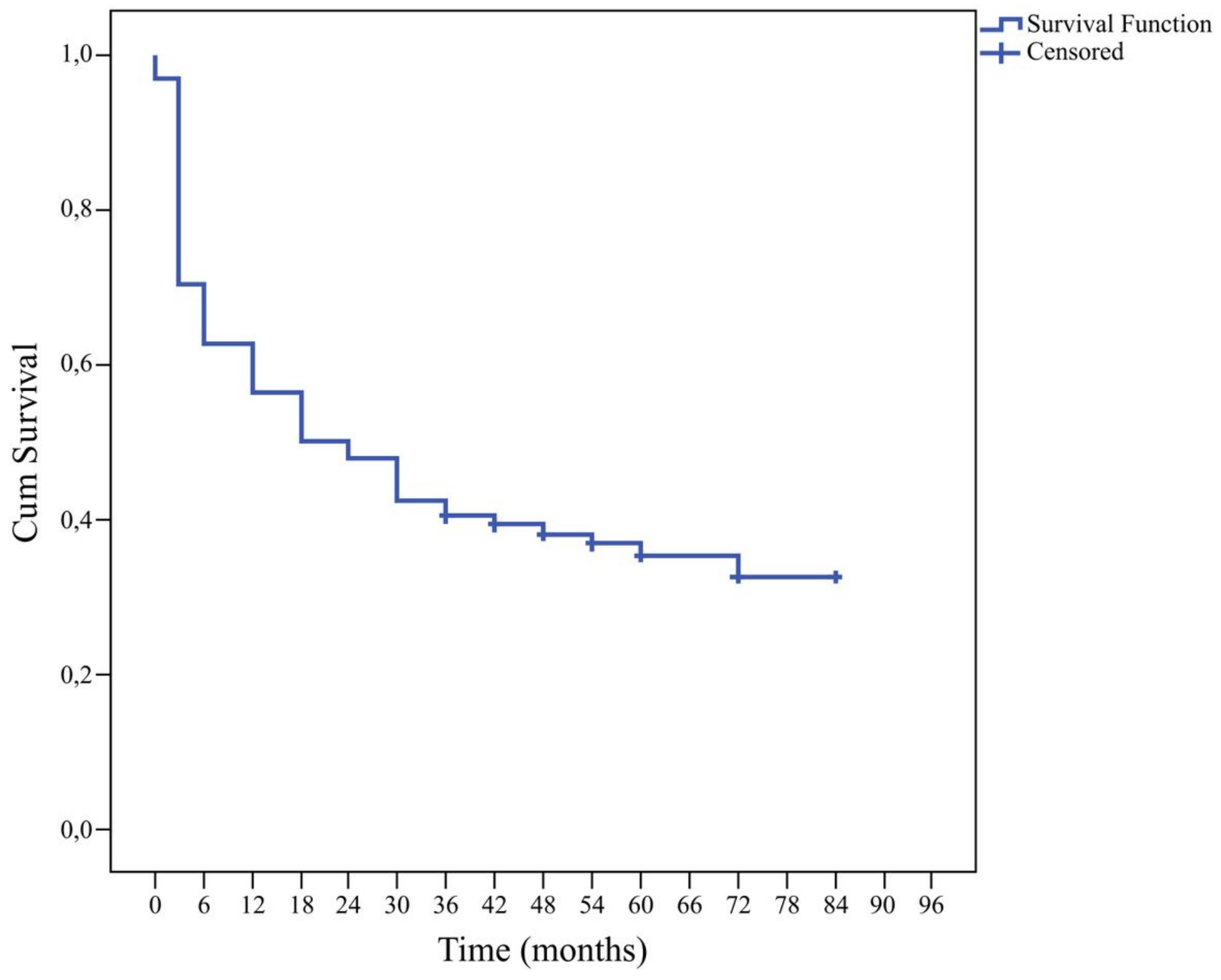

Figure 1

Time of the onset of any symptom of late UGT grade $\geq 1$ After the end of RT, patients were followed up and symptoms of late UGT grade $\geq 1$ were estimated every 6 months until 96 months. The estimated median time of the onset of symptoms of late UGT grade $\geq 1$ is 18 months 


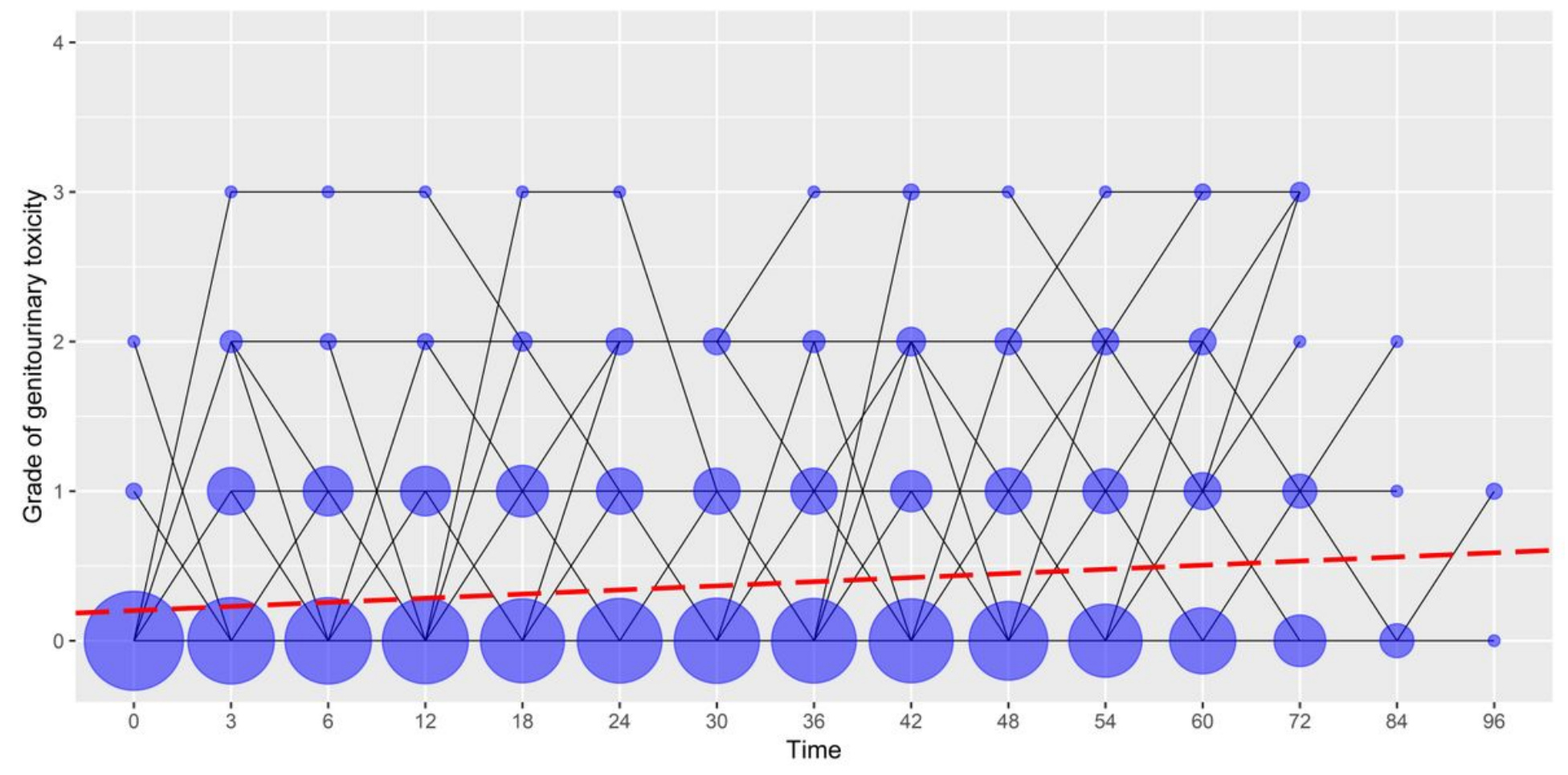

Figure 2

Changes in the degree of late UGT at each time point for each patient Changes in the late genitourinary toxicity grades at specific time points: 0 -check-up during the first week of RT, check-up at $3,6,12,18,24,30,36,42,48,54,60,72,84,96$ months after the completion of radiation treatment. The lines represent changes in the degree of late urogenital toxicity at particular time points for each patient. Circle area is proportional to the number of patients. In the pre-treatment point it is $100 \%$. At each time point, the sum of the circle area is $100 \%$. 